\title{
Quasi-Newton Power Flow Using Partial Jacobian Updates
}

\author{
Adam Semlyen, Life Fellow, IEEE and Francisco de León
}

\begin{abstract}
We present a quasi-Newton power flow methodology that incorporates several strategies to obtain substantial computing savings. Newton steps are combined with constant Jacobian (or "simple") steps and partial Jacobian updates to get an efficient quasi-Newton method. The methodology proposed includes the possibility of selecting the next best step by measuring the residuals. Partial Jacobian Updates (PJU) are included in the quasi-Newton power flow using $\mathrm{LU}$ factorization updates and/or the Matrix Modification Lemma. The method has been tested with systems ranging in size from 14 to 6372 buses. For large power systems we have obtained savings (in flops) in the order of $\mathbf{5 0 \%}$ compared to Newton's method.
\end{abstract}

Index Terms-Matrix modification lemma, matrix refactorization, Newton power flow, partial Jacobian updates.

\section{INTRODUCTION}

$\mathbf{T}$ HE POWER flow problem is still commonly solved (in 2000) using the Fast Decoupled Power Flow (FDPF) method proposed by Stott and Alsaç in 1974 [1]. Often, however, the FDPF fails to converge when the power system is close to its operating limits and when the $\mathrm{R} / \mathrm{X}$ ratios of the transmission lines are high. The latter is typical for longitudinal systems of developing countries. The next logical choice is to use the Newton Power Flow (NPF) as described by Tinney and Hart in 1967 [2]. Although NPF has excellent convergence properties even under stressed circumstances, the Jacobian matrix needs to be factorized at every iteration. Therefore, the NPF solution is expensive.

One strategy used in this paper to reduce the computational burden of a NPF solution is to keep the Jacobian constant for a number of iterations. Although the idea is certainly not new and very likely has been explored before, we do not know of published results. When the Newton Method was described in [2], the authors also noted that the use of a constant Jacobian was possible. The conclusions of the study (using very limited computing resources and small systems) showed that the c.p.u. savings were not significant. The Jacobian has been held constant for the study of transient stability and the name of (Very) Dishonest Newton [9] has been used. Those studies are aimed for the real-time simulation of stability using computers with multiprocessors.

Manuscript received December 15, 1999. This work was supported by the Natural Sciences and Engineering Research Council of Canada.

A. Semlyen is with the Department of Electrical and Computer Engineering, University of Toronto, Toronto, Ontario, Canada M5S 3G4 (e-mail: semlyen@ecf.utoronto.ca).

F. de León is on leave at the University of Toronto. He is with the Instituto Politécnico Nacional, Escuela Superior de Ingeniería Mecánica y Eléctrica, 07738 - México, D.F., México (e-mail: fdeleongm@ @otmail.com) .

Publisher Item Identifier S 0885-8950(01)04528-X
An idea introduced in this paper to reduce the computing time is the partial update of the Jacobian matrix. Since not all equations converge together, there is no need for updating the whole Jacobian at every step. For example, at a certain step in the iteration process we commonly find that: a) some residuals have already converged; b) most residuals are decaying fast enough to converge with a constant Jacobian within a few iterations; and c) a few (identifiable) residuals will converge fast only if the corresponding part (rows) of the Jacobian is updated.

The Quasi-Newton Power Flow (QNPF) proposed in this paper exploits the combination of: a) Newton steps where the whole Jacobian is computed and consequently refactorized; b) Simple steps where the most recent Jacobian is reused with forward and backward substitutions; c) Partial Jacobian updates where only a small portion of the Jacobian matrix is updated. Partial Jacobian updates are introduced in the power flow program in two ways: 1) Partial LU refactorization of the Jacobian; and 2) A simple step solution is corrected using the Matrix Modification Lemma (MML).

The objective of this paper is to efficiently solve the load flow problem retaining the robust convergence characteristics of Newton's method. Although the convergence of our QNPF method is not quadratic (as with the NPF) we have observed that the method always converged when the NPF converged. Our results are comparable with those obtained using full Newton combined with GMRES solvers [3].

We have focused our attention on the way the Jacobian is updated and factorized since its factorization accounts for approximately $85 \%$ of the cpu time. We propose an adaptive technique based on the evolution of the residuals that allows the identification of the best next step. For example, at some point may be more convenient to factorize the whole Jacobian than to keep it constant, while at some other stages a partial update (and corresponding partial refactorization) may be more convenient. Partial refactorizations are typically used in contingency analysis and have been used in the Newton power flow to deal with transformer taps and other automatic adjustments [6], but they have not been applied with a local identification strategy for the convergence trajectories.

\section{Characterization OF QNPF}

\section{A. Newton Power Flow}

The linearized Newton power flow equations are of the form

$$
J \Delta x=b
$$

where

$\Delta x$ corresponds to the increments in the variables (voltage magnitudes and power angles); 


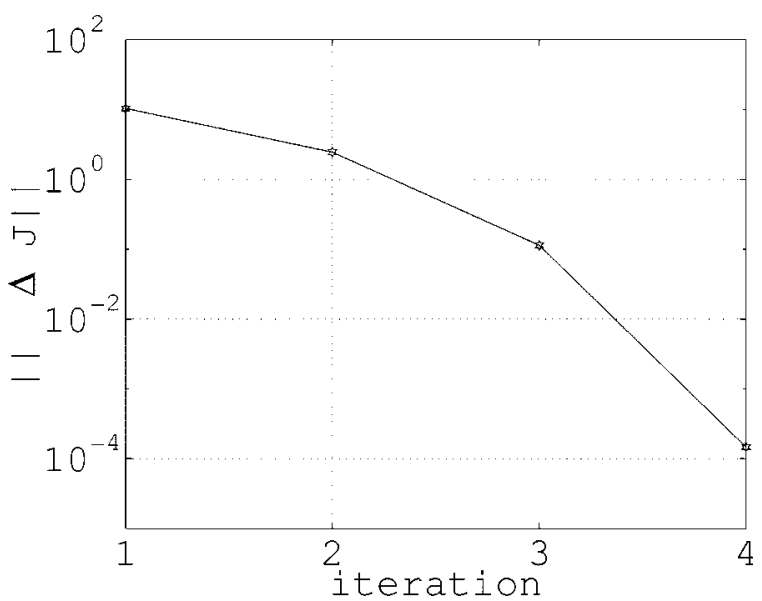

Fig. 1. Infinity norm of the difference between the current and converged Jacobians.

$b \quad$ represents the (active and reactive) power mismatches; $J \quad$ Jacobian matrix.

We have used the formulation described in [4] for the elements of $J$ since it offers an efficient computation scheme.

The local quadratic convergence property of Newton's method is reviewed in the simple analysis presented for a single nonlinear equation in Appendix I.

The Jacobian of a power flow problem is a real, sparse, nonsymmetric, positive definite matrix with a large condition number that increases with the size of the system. The condition number for the IEEE 14 bus test system is $1.1 \times 10^{2}$, for the 118 bus test system it is about $3 \times 10^{3}$, and for our 3186 bus test system it is $5.6 \times 10^{4}$.

All results reported in this section are based on the IEEE118 bus test system, however similar conclusions can be drawn from all the other test systems used in this paper. We note that the elements of the Jacobian and its spectrum do not vary substantially from step to step in the Newton method. In Fig. 1, we show the infinity norm $\|\Delta J\|_{\infty}$ of the difference between the current and the converged Jacobians.

We see that the changes occur in the Jacobian elements mainly in the first steps. After only four steps the changes in the elements are smaller than $10^{-3}$ in actual value and even less in relative value. Therefore, reusing the Jacobian for one or more steps seems to be reasonable. Moreover, it is more convenient to update the Jacobian at the earlier steps than toward the end of the iterative cycle.

In Fig. 2, we show the spectra of the first Jacobian (flat start) and of the converged Jacobian. Because the Jacobian matrix is nonsymmetric, its spectrum has real and imaginary eigenvalues. We have been able to use a semilog scale since the Jacobian is positive definite, and so we can observe the changes in both the small and large eigenvalues. It is interesting to note that, as expected, the spectrum of the Jacobian does not change significantly from step to step during the iterations of Newton's method. In particular we note some change in the eigenvalue distribution at the central part of the spectrum. The large and small eigenvalues remain virtually unchanged.

Fig. 3 shows the variation of the 181 residuals or mismatches (corresponding to $\Delta P$ and $\Delta Q$ ) for our 118 bus test system.
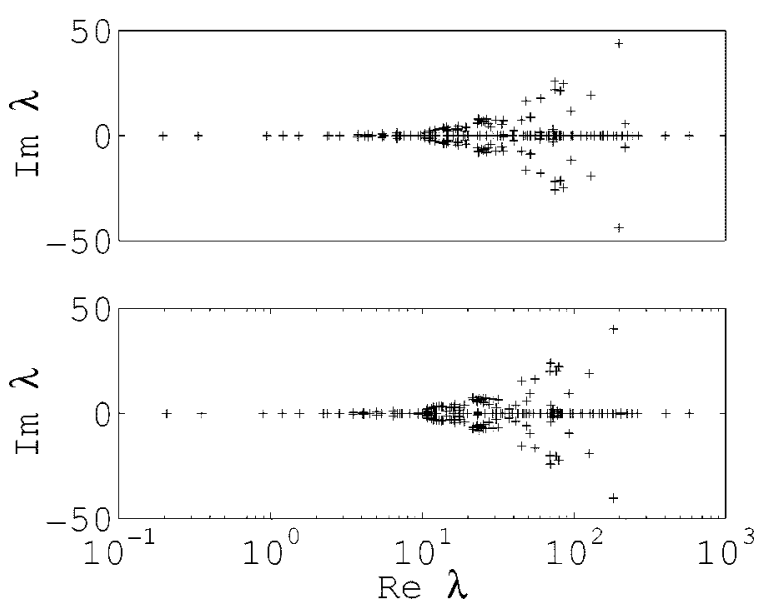

Fig. 2. Spectra of the first and converged Jacobians.

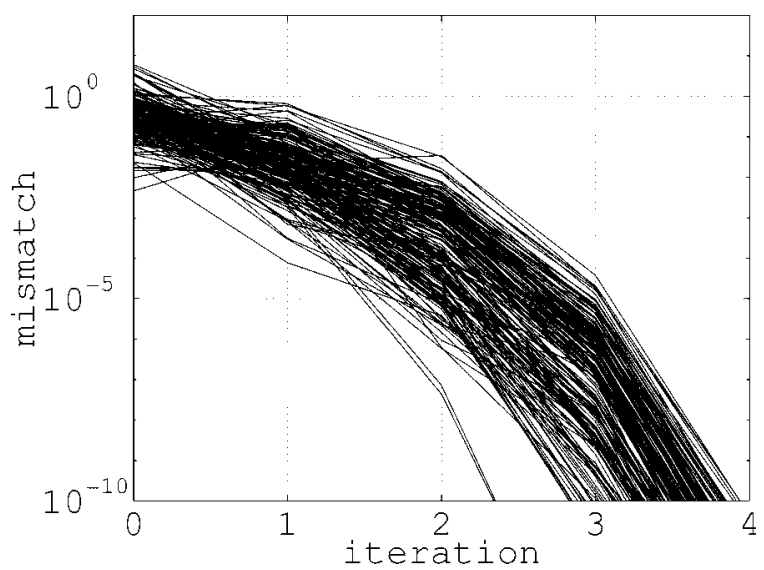

Fig. 3. Residuals (or mismatches) for the IEEE 118 bus test system. We have 181 equations.

We make the following important remarks: a) Although a few mismatches converge very fast (in 2 or 3 iterations depending on the tolerance) most mismatches converge in a bundle with approximately the same rate, but with a different residual value; b) In general the convergence trajectories do not cross each other and their slope becomes steeper (more negative) with every Newton step; c) The last Newton step greatly overshoots the specified tolerance of $10^{--5}$. We could save computing effort by keeping the last Jacobian constant (this behavior will be exploited below); d) The thickness of the bundle of convergence trajectories is very wide toward the end of the iterative cycle (more than 6 orders of magnitude!). For example, at iteration 3 most equations have already converged, but because the stopping criterion is that all mismatches should be below the tolerance we are "unnecessarily" computing (and therefore factorizing) parts of the Jacobian. This characteristic behavior will be exploited below to save computing time using partial Jacobian updates.

The quadratic convergence characteristics of Newton's method can be observed in the mismatches, in the errors and even in the Jacobian matrix itself.

When plotting errors $\varepsilon_{k}=\left|x_{k}-x_{\infty}\right|$ we can make identical observations as with mismatches (results are not shown due to space limitations). In Appendix II, we show that the convergence of the Jacobian matrix is also quadratical since its 


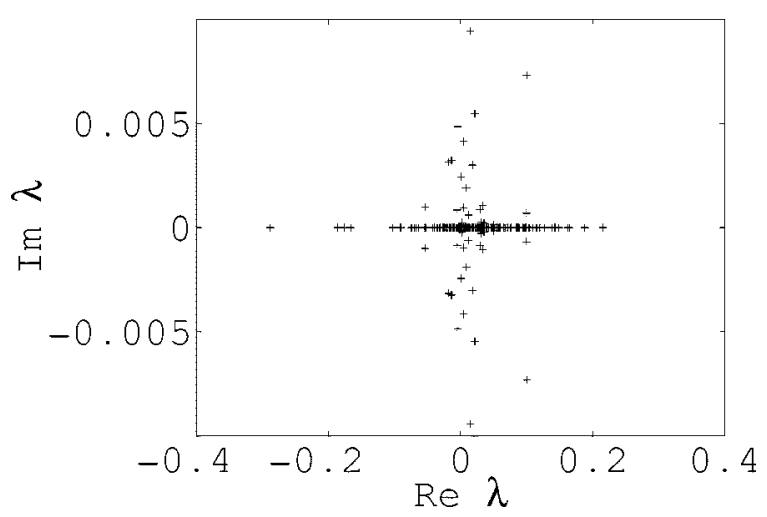

Fig. 4. Spectrum of the iteration matrices.

variations are proportional to the increments in the variables (or in the mismatches); See Fig. 1.

\section{B. Constant Jacobian}

As shown in the previous section, the Jacobian matrix remains almost constant, suggesting that a good approximation would be to keep the Jacobian constant throughout the iterative process.

Iteration Matrix: The convergence properties of a quasiNewton iterative method can be estimated using an iteration matrix [5]. The iteration matrix relates the new value of a variable to its previous value. In Appendix III we present a derivation of the iteration matrices $M_{\varepsilon}$ for errors and $M_{\eta}$ for mismatches for a constant Jacobian iterative method. The iteration matrices are:

$$
M_{\varepsilon}=I-J_{0}^{-1} J ; \quad M_{\eta}=I-J J_{0}^{-1}
$$

where $J_{0}$ is an approximation of the exact Jacobian $J$.

The spectrum of an iteration matrix gives an indication of the convergence of the method. For example, when all eigenvalues are smaller than one, the quasi-Newton method will converge (if Newton itself converges). If at least one eigenvalue is equal or larger than one, then the quasi-Newton method fails. The dominant eigenvalue (the one with the largest magnitude) determines the convergence rate. Quadratic convergence is only possible when using the exact Jacobian so that all eigenvalues of the iteration matrices are zero.

Note that both iteration matrices, $M_{\varepsilon}$ and $M_{\eta}$, have the same eigenvalues since $M_{\eta}=J M_{\varepsilon} J^{-1}$. In Fig. 4 we show their spectrum for an iterative process with the first Jacobian held constant for the 118 bus test system. We observe that the eigenvalues are almost real, indicating that the iteration matrices are virtually symmetrical. The module of the dominant eigenvalue is 0.3312 . It characterizes the asymptotic rate of convergence.

In Fig. 5 we show the variation of the corresponding 181 residuals. We note that the convergence rate is linear. As expected, we found that the eigenvector of $M_{\eta}$ corresponding to the dominant eigenvalue points to the slowest converging mismatches.

We note from Fig. 5 that: a) The method needs more iterations (than full Newton) to reach converge; b) The convergence bundle widens just slightly as the iterations advance; c) Some convergence trajectories cross each other; d) The overall slope remains constant after the second iteration.

We have tested a number of constant matrix solution strategies. For example, we took one by one the simplifications used

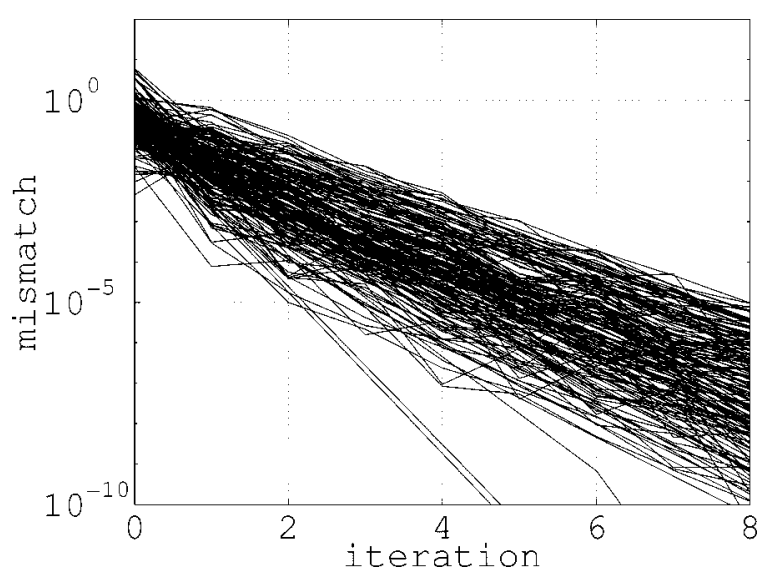

Fig. 5. Residuals when using a constant Jacobian.

to obtain the Fast Decoupled Power Flow as described in [1]. When we neglect the coupling submatrices of the constant (first) Jacobian, the spectrum of the iteration matrix presents many complex conjugate eigenvalues (the matrix is nonsymmetrical) and the dominant eigenvalue becomes 0.4144 . By neglecting the conductance, the dominant eigenvalue worsens to 0.7734 . It is interesting to note that a compensation effect is produced when the shunt reactances are omitted in the $P-\Delta \theta$ equation: the dominant eigenvalue reduces to 0.4176 . Finally, when we neglect the resistance of the lines in the $P-\Delta \theta$ equation, the dominant eigenvalue of the iteration matrix for the FDPF is 0.4330 . Note that for the FDPF the eigenvalue distribution does not by itself determine the convergence characteristics of the method. One should be aware that the approximations made in one of the submatrices compensate for the approximations made in the other; see [7] for further details.

\section{Solution Strategies}

1) Full Newton: As mentioned before, there are situations where the FDPF fails to converge. Thus, we need to use a method with better convergence properties such as the NPF or a QNPF.

The full Newton method offers the best possible convergence rate (viz., quadratic). However, because for every (Newton) step we need to refactorize the Jacobian matrix, the method is expensive in terms of computing time.

Since nowadays computers have become increasingly more powerful, engineers look also at much larger problems. As the size of the system increases, the floating point operations (flops) of LU complete factorizations become very expensive even for the solution of sparse systems. Therefore, the efficiency issue remains very important.

2) Number of Simple Steps: One strategy suggested in [2] to reduce the computing time is to keep the Jacobian constant for a number of iterations. We will call these iterations simple steps since they consist basically in forward and backward substitutions (there is no factorization involved as in Newton steps). Tinney and Hart [2] experimented including one and two simple steps between Newton steps with relatively small systems of 286 and 443 buses.

We have made a considerable number of experiments with combinations of Newton and simple steps using many systems. 
Our guidelines for experimenting included the knowledge gained above regarding the facts that: a) The largest variations in the Jacobian occur in the first steps; and b) While Newton steps are expensive and tend to converge quadratically, simple steps are inexpensive but converge linearly.

Considering the above remarks we tested two sets of constant Jacobian strategies: a1) One Newton step followed by enough simple steps to obtain convergence; a2) Two consecutive Newton steps followed by simple steps only; a3) Three consecutive Newton steps and then simple steps only, and so on; b1) Sequences of one Newton step and one simple step; b2) Trains of one Newton step followed by two simple steps; b3) Series of one Newton step followed by three simple steps, and so on.

Although with the above sets of experiments we were in general able to obtain substantial savings in the number of flops, the best strategy is very much problem dependent.

3) Proposed Strategy: We propose a strategy with decision to be taken as to the use of a Newton step or a simple step according to the predicted value of the largest mismatch. At this point, we focus on the largest mismatch only because it determines when the method has converged.

We predict (based on the latest two computed steps) if a Newton step or $m$ simple steps would yield a smaller mismatch. $m$ is the number of simple steps that cost (in flops) the same as a Newton step.

The convergence rate of Newton's method is quadratic only when we approach the solution. At intermediate steps the convergence is faster than linear, but slower than quadratic (it is super-linear). Thus the mismatch $\eta$ for the next Newton step can be estimated as

$$
\eta_{k+1}=\alpha \eta_{k}^{\beta}
$$

(with $1<\beta<2$ ) where $\alpha$ is predicted form (3) using the previous two steps, as

$$
\alpha=\frac{\eta_{k}}{\eta_{k-1}^{\beta}}
$$

When $\eta$ is large (for the first steps), $\beta \approx 1$, and when $\eta$ is small (for the last steps), then $\beta \approx 2$. Thus we propose

$$
\beta=2-\frac{\eta_{k}}{\eta_{k-1}} \text {. }
$$

When $\eta_{k-1} \approx \eta_{k} \rightarrow \beta \approx 1$ and when $\eta_{k-1} \gg \eta_{k} \rightarrow \beta \approx 2$.

The convergence rate of the simple steps is only linear,

$$
\eta_{k+1}=K \eta_{k}
$$

and the estimated mismatch $\mathrm{m}$ steps ahead is

$$
\eta_{k+m}=K^{m} \eta_{k}
$$

where $K$ is calculated using the previous two mismatches

$$
K=\frac{\eta_{k}}{\eta_{k-1}} .
$$

The process starts with a Newton step followed by a simple step. After every simple step we compare the predicted mismatches obtained from equations (3) and (7). If (7) predicts a smaller value we continue with a simple step. Note that $\alpha$ and $\beta$ are not updated after a simple step, but only after a Newton

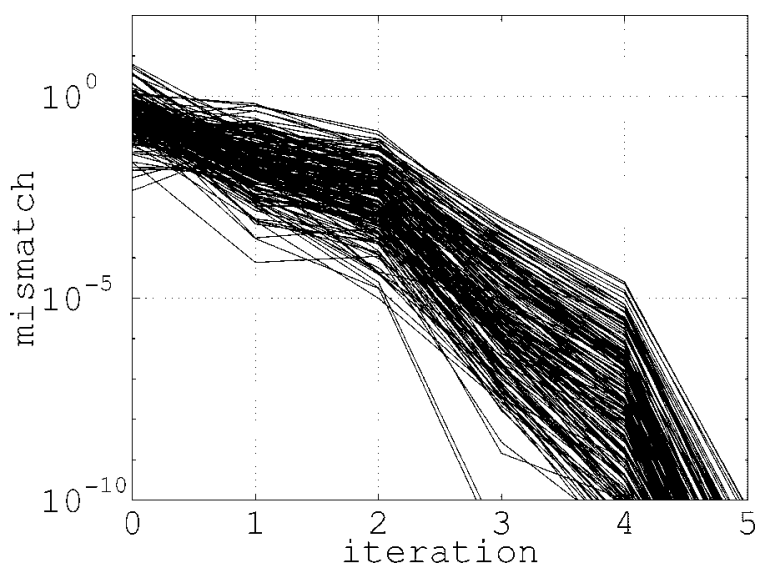

Fig. 6. Residuals using the proposed strategy that combines Newton and simple steps.

step. However, the new computed $\eta$ is used in (3) to predict the next Newton mismatch. When (3) predicts a smaller mismatch than (7) a Newton step is applied which is followed by a simple step.

In Fig. 6, we show the convergence trajectories for the residuals (mismatches) for the 118 bus test system when the strategy is implemented. We make the following remarks: a) The proposed method converges in more iterations than Newton, but fewer than with the constant Jacobian strategy; b) The thickness of the convergence bundle increases considerably, for example, by the fourth iteration it is almost 9 orders of magnitude wide; c) The convergence trajectories in general do not cross each other; d) The last (Newton) step greatly overshoots the specified tolerance of $10^{-5}$; e) When we apply a Newton step the slope increases while for a simple step the slope remains the same.

\section{PARTIAL Jacobian Updates}

Further savings are obtained by including Partial Jacobian Updates (PJU) in the solution strategy. We have noticed that all strategies share the feature that after a few steps many equations have already converged while some others may be well behind. Furthermore, we have noticed that for a fairly large number of equations (corresponding to the convergence trajectories) it is not necessary to update the Jacobian to get to convergence in a few simple steps. However, the trajectories that determine the convergence of the whole method (the ones at the top) will take a great number of simple steps to reach convergence. Therefore, we recompute the rows of the Jacobian for the slowly converging equations while keeping constant the greatest part of the matrix.

\section{A. Laggards}

We call "laggard" a residual (or equation) with the most slowly converging trajectory, or, by extension, the trajectory at the top of the bundle. Typically, we can identify more than one laggard during the solution process.

1) Heavy Loads: The laggard residuals are identified easily when the system has one (or a few) overloaded buses. We have found that the laggards correspond to the $\Delta P$ and/or $\Delta Q$ of the overloaded buses and their first neighbors. They are also associated with buses connected through weak links including again 
the neighboring buses directly connected to them. This identification becomes important when we are performing voltage stability studies. Thus, we are capable of separating the equations of those buses from the beginning and apply standard partial refactorization techniques. For example, before applying the optimal ordering for factorization purposes, the laggard equations are moved to the bottom of the matrix. We factorize the whole matrix once or perhaps twice depending on the nature of the problem. Subsequent Jacobian updates and the associated factorizations are only performed to the laggard equations.

2) Residuals: It is not always possible to identify the laggard equations before starting the process. However, in general the laggard residuals may be identified numerically after a few steps in the iterative process. Typically, the laggards will appear during the iterative process after the first two steps. In our proposed methodology they could be conveniently identified after the initial Newton and simple steps. On few occasions the laggards will only show up toward the end of the process.

\section{B. Refactorization by LU Update}

The preferred alternative to including the PJU into the iterative process is updating the LU factorization for the constant Jacobian to reflect the changes of the partially updated Jacobian. Here we present an efficient procedure for finding

$$
A=L U
$$

when a large part $A_{11}$ of $A$ has a known factorization. Let (9) be partitioned as:

$$
\left[\begin{array}{ll}
A_{11} & A_{12} \\
A_{21} & A_{22}
\end{array}\right]=\left[\begin{array}{cc}
L_{1} & 0 \\
M & L_{2}
\end{array}\right]\left[\begin{array}{cc}
U_{1} & N \\
0 & U_{2}
\end{array}\right]
$$

where the factorization of $A_{11}$ is given by:

$$
A_{11}=L_{11} U_{11} \text {. }
$$

$A_{11}$ is a of order $n \times n$ and $A_{22}$ of order $p \times p$. Since $p$ represents the number of laggard equations that need a PJU, $n \gg p$. We also note that: $L_{1}, L_{2}$ are lower triangular matrices; $U_{1}, U_{2}$ are upper triangular matrices; and $M, N$ are rectangular matrices. Developing (10) we find:

$$
\begin{aligned}
& L_{1}=L_{11}, \quad U_{1}=U_{11}, \quad N=L_{1} \backslash A_{12}, \quad M=A_{22} / U_{1} \\
& {\left[L_{2}, U_{2}\right]=\mathrm{LU}\left(A_{22}-M N\right) .}
\end{aligned}
$$

The last three equations are written in quasi-Matlab code and simply mean that: $N$ is obtained from the solution of $L_{1} N=$ $A_{12} ; M$ is obtained from $M U_{1}=A_{21}$; and $L_{2}$ and $U_{2}$ are obtained from the LU factorization of the matrix $A_{22}-M N$. Thus, we have all the submatrices that compose equation (11) by inexpensive computations since $p$ is a small number, 4 in our next example.

In Fig. 7, we show the residuals for the same case (118 bus test system with the proposed strategy) when we apply the PJU and LU update at step 4. Many trajectories have been removed from the figure for clarity. We get to the specified convergence tolerance $10^{-5}$ in only four iterations. The steps are as follows:

- We start with a Newton step followed by a simple step;

- The differences commence with the second Newton step since we have to separate the portion of the Jacobian matrix that will be updated later; In our example,

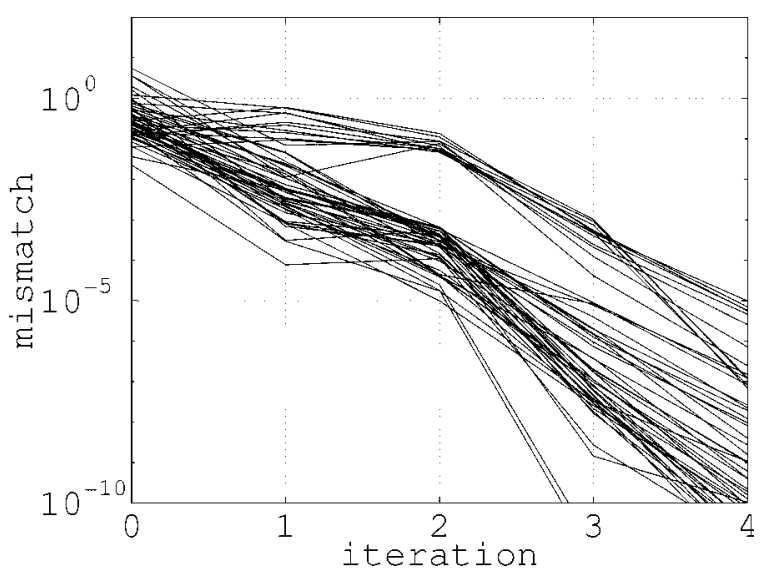

Fig. 7. Partial Jacobian Update applied at step 4.

the worst four equations are left at the end of the matrix, but the whole matrix has to be factorized because this separation step is also a Newton step;

- The final step consists of two stages: a) The PJU of only four equations and using (12) for computing the new LU factors; and b) Forward and backward substitutions to compute the new state variables (and residuals).

The following remarks are important: a) Up to step number three the behavior is identical to the case of Fig. 6; b) For the last step the four partially updated equations cross the bundle since their slope is characteristic of a Newton step and the other 177 equations exhibit the behavior of a simple step. In this case we have saved just about a full Newton step, however for larger systems we obtain much larger savings (see Section IV).

\section{Using the Matrix Modification Lemma}

The well know Matrix Modification Lemma (MML) can also be used to incorporate the PJU into the iterative process. The MML is a tool that allows to obtain the solution of a set of equations when we already have the solution of "almost" the same set of equations, the difference being a low rank modification of the coefficient matrix. It is commonly used in power systems for contingency analysis (when only a few network changes occur) because it makes unnecessary the refactorization of the network matrix; see Appendix IV. An MML step can be seen as a correcting step since we first compute a simple step as a predictor. Later, when it is necessary, we perform a PJU and correct the solution obtained from the simple step using MML. Only the solution is updated, the actual Jacobian matrix and its associated LU factors are not changed.

Fig. 7 also shows the residuals for the PJU using MML. The results are, as expected, identical to the LU update case since both methods solve the same set of equations. As before, we also start with a Newton step, followed by a simple step and another Newton step. Finally, the following simple step is corrected with MML when the PJU has been applied to only the worst four residuals (or equations).

\section{When to Use MML or LU Update}

Both methodologies, MML or LU update, give the same numerical results. However, there are important differences in their 


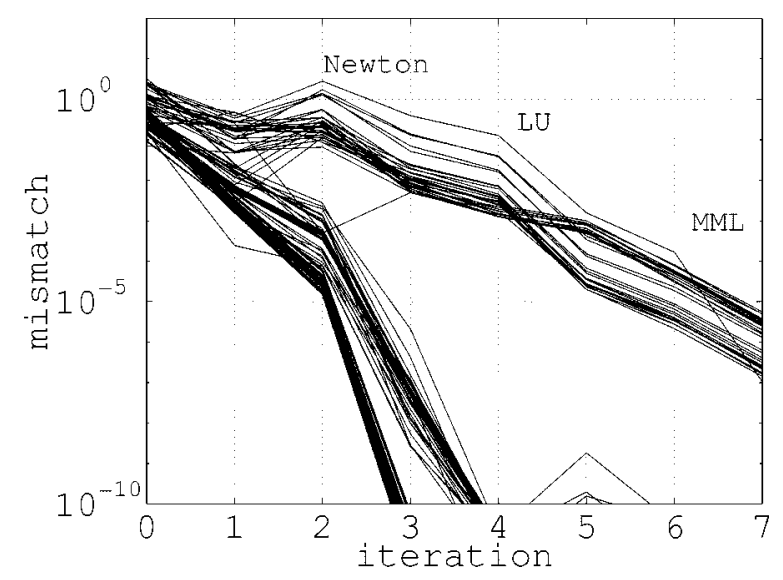

Fig. 8. Extreme residuals for the 3186 bus system, showing the application of PJU with LU update and MML.

application for the solution of the nonlinear equations. While an LU update could be seen as a definite step (since the triangular factors are modified), MML is simply a correcting step.

When we need several PJU steps, or even simple steps after a PJU has been applied, the LU update is less expensive than the MML. However, since the application of LU updates requires a Newton separation step, it is only useful when the laggards can be identified early on. The identification can be done using not only the value of the mismatches but their slope as well.

On the other hand, for the last few steps (one or two), an MML is more attractive. For example, a good application is when more than $99 \%$ of equations have already converged. MML is best when after a simple step we did not get to full convergence because of a few laggard mismatches. Thus, these laggards can be "pushed down" with PJU using MML, and we get convergence immediately.

The best results are obtained when we combine PJU steps with LU updates with MML, especially in very large systems. For the residuals (or equations) corresponding to overloaded buses and those that are identified by the second step we apply (one or more) PJU steps with LU updates. For some other difficult residuals that appear during the iterative cycle (that could not be identified early but slow down the convergence), we apply PJU with MML to force them to converge more quickly. This is illustrated in Fig. 8 (more than 4000 central residuals are not shown for better visualization). At step 5 we apply a PJU with LU update to 10 equations and at step 7 a PJU with MML to only one equation.

We have not explored every possibility to improve the efficiency of the QNPF. For example, in [6] several refactorization techniques are examined. Among them we selected the ones easier to implement in Matlab. In a production grade power flow program one could improve the procedure by selecting a different refactorization technique. Another refinement is to use the approach described in [8]. The power mismatches are set to zero when they are below certain tolerance, thus saving further evaluation. While for the FDPF the savings are substantial, in our quasi-Newton approach the cpu savings would be relatively small since the factorization of the Jacobian consumes by far the most cpu time. It has also been suggested in the discussions of [8] that substantial savings can be obtained by the use of the most appropriate convergence tolerance for each node individually: uniform significance of the MW and Mvar mismatches is achieved by considering the voltage level and the specified power at each node.

\section{Test Results}

\section{A. Systems Used for Testing}

Several systems have been used for testing the methodologies described in this paper. We used the standard IEEE test systems of $14,24,39$, and 118 buses. We built several large systems by placing 118 bus test systems in a matrix; we added connections between neighboring buses and superimposed a high voltage grid to reflect reality. Thus we obtained test systems of 354, 1062, 3186 and 6372 buses. In all cases, we have used a flat start, i.e., all voltage magnitudes equal to 1.0 p.u. (except for the specified voltage buses) and all power angles equal to zero. We have changed the loading conditions at certain buses to observe the effect of the load on the residuals.

\section{B. Comparison}

We have used the number of flops rather than c.p.u. time as the measurement of efficiency of an algorithm since c.p.u. time is very much dependent on human and machine factors. Flops, on the other hand, are only dependent on human factors and to a lesser extent when using a good compiler.

Tables I and II summarize the numerical results. We have used two tolerances, one of practical value for power flow studies equal to $10^{-5}$ (results reported in Table I). The other, a high precision convergence tolerance equal to $10^{-12}$ (Table II), serves mainly for observing the conversion characteristics of the quasiNewton method. The latter may be also relevant in applications outside the analysis of power systems.

The guidelines for experimentation regarding the use of Newton or simple steps were discussed in Section II-C-2. In Section III-D we discussed the use of PJU with LU update and MML. The figures in the tables correspond to the number of mega-flops (Mflops). $N$ indicates that the results correspond to Newton's method. A hyphen is used when we have not achieved improvements with PJU and therefore the case is not practical. In Fig. 9 we show the main results from Table I by graphically comparing the different methods against Newton.

From the results we make the following remarks: a) For small systems the savings (with any strategy) are small; b) Using Newton's method we can go from a convergence tolerance of $10^{-5}$ to $10^{-12}$ with only $20 \%$ more of computing effort (this is consistent with its quadratic convergence characteristics); c) Comparing strategies " $a$ " and " $b$ " we note that in general b's are better than a's; d) The proposed strategy (in Section II-C-3, labeled OP in the tables and Fig. 9) is consistently competitive with the best of strategy "a" or "b." The savings increase with the size of the system and they are from $30 \%$ to $40 \%$ for large systems; e) For the high accuracy case $\left(10^{-12}\right)$ there are no savings by including the PJU since very many mismatches need to be updated and therefore a Newton step is more adequate; f) For the practical accuracy of $10^{-5}$ the PJU with LU update helps with another $10 \%$ to $20 \%$, thus the total flop savings are 
TABLE I

Mflops For Different StRategies When THE CONVERGENCE TOLERANCE IS $10^{-5}$

\begin{tabular}{c|c|c|c|c|c|c|c|c|c|c|c|c|c}
\hline System & $\mathrm{N}$ & $\mathrm{a} 1$ & $\mathrm{a} 2$ & $\mathrm{a} 3$ & $\mathrm{a} 4$ & $\mathrm{a} 5$ & $\mathrm{~b} 1$ & $\mathrm{~b} 2$ & $\mathrm{~b} 3$ & $\mathrm{~b} 4$ & $\mathrm{OP}$ & $\mathrm{LU}$ & $\mathrm{MML}$ \\
\hline 14 & 0.0111 & 0.0130 & 0.0104 & 0.0111 & $\mathrm{~N}$ & $\mathrm{~N}$ & 0.0104 & 0.0103 & 0.0115 & 0.0126 & 0.0104 & - & - \\
\hline 24 & 0.0268 & 0.2114 & 0.0277 & 0.0231 & $\mathrm{~N}$ & $\mathrm{~N}$ & 0.0272 & 0.0293 & 0.0334 & 0.0375 & 0.0273 & - & - \\
\hline 39 & 0.0471 & 0.0617 & 0.0403 & 0.0402 & $\mathrm{~N}$ & $\mathrm{~N}$ & 0.0436 & 0.0368 & 0.0403 & 0.0438 & 0.0436 & - & - \\
\hline 118 & 0.1320 & 0.1196 & 0.1173 & 0.1143 & $\mathrm{~N}$ & $\mathrm{~N}$ & 0.1246 & 0.1067 & 0.1066 & 0.1169 & 0.1246 & 0.1107 & - \\
\hline 354 & 0.4408 & 0.4442 & 0.4127 & 0.3780 & $\mathrm{~N}$ & $\mathrm{~N}$ & 0.4102 & 0.3469 & 0.3466 & 0.3787 & 0.4102 & 0.3538 & - \\
\hline 1062 & 2.0472 & 2.1945 & 2.2805 & 1.8198 & 1.8812 & $\mathrm{~N}$ & 1.5048 & 1.7123 & 1.4403 & 1.5432 & 1.5049 & 1.4123 & 1.3914 \\
\hline 3186 & 17.521 & 12.806 & 15.428 & 13.993 & 13.626 & 15.040 & 12.909 & 10.778 & 11.129 & 8.999 & 11.140 & 7.8712 & 7.2041 \\
\hline 6372 & 32.773 & 30.086 & 35.120 & 30.421 & 27.867 & 28.890 & 24.279 & 21.099 & 21.801 & 22.503 & 23.257 & 17.454 & 16.521 \\
\hline
\end{tabular}

TABLE II

MFLOPS FOR DifFERENT STRATEGIES WHEN THE CONVERGENCE TOLERANCE IS 10-12

\begin{tabular}{c|c|c|c|c|c|c|c|c|c|c|c|c|c}
\hline System & $\mathrm{N}$ & $\mathrm{a} 1$ & $\mathrm{a} 2$ & $\mathrm{a} 3$ & $\mathrm{a} 4$ & $\mathrm{a} 5$ & $\mathrm{~b} 1$ & $\mathrm{~b} 2$ & $\mathrm{~b} 3$ & $\mathrm{~b} 4$ & $\mathrm{OP}$ & $\mathrm{LU}$ & $\mathrm{MML}$ \\
\hline 14 & 0.0142 & 0.0256 & 0.0163 & 0.0135 & $\mathrm{~N}$ & $\mathrm{~N}$ & 0.0135 & 0.0158 & 0.0138 & 0.0150 & 0.0135 & - & - \\
\hline 24 & 0.0324 & 0.2114 & 0.0483 & 0.0314 & 0.0309 & $\mathrm{~N}$ & 0.0350 & 0.0334 & 0.0375 & 0.0396 & 0.0350 & - & - \\
\hline 39 & 0.0575 & 0.1216 & 0.0614 & 0.0472 & 0.0506 & $\mathrm{~N}$ & 0.0471 & 0.0507 & 0.0577 & 0.0508 & 0.0471 & - & - \\
\hline 118 & 0.1601 & 0.2428 & 0.1795 & 0.1454 & 0.1424 & $\mathrm{~N}$ & 0.1350 & 0.1452 & 0.1378 & 0.1377 & 0.1350 & - & - \\
\hline 354 & 0.5363 & 0.8625 & 0.6405 & 0.5082 & 0.4734 & $\mathrm{~N}$ & 0.4427 & 0.4749 & 0.4442 & 0.4439 & 0.4428 & - & - \\
\hline 1062 & 2.4221 & 4.2526 & 3.9513 & 2.7597 & 2.1945 & 2.2561 & 1.9842 & 1.8167 & 1.9197 & 2.1269 & 1.9843 & - & - \\
\hline 3186 & 20.359 & 24.382 & 27.174 & 20.400 & 16.474 & 16.463 & 13.265 & 13.972 & 11.485 & 11.836 & 11.851 & - & - \\
\hline 6372 & 38.086 & 59.577 & 64.434 & 49.725 & 36.447 & 33.179 & 30.307 & 26.412 & 23.231 & 23.933 & 24.687 & - & - \\
\hline
\end{tabular}

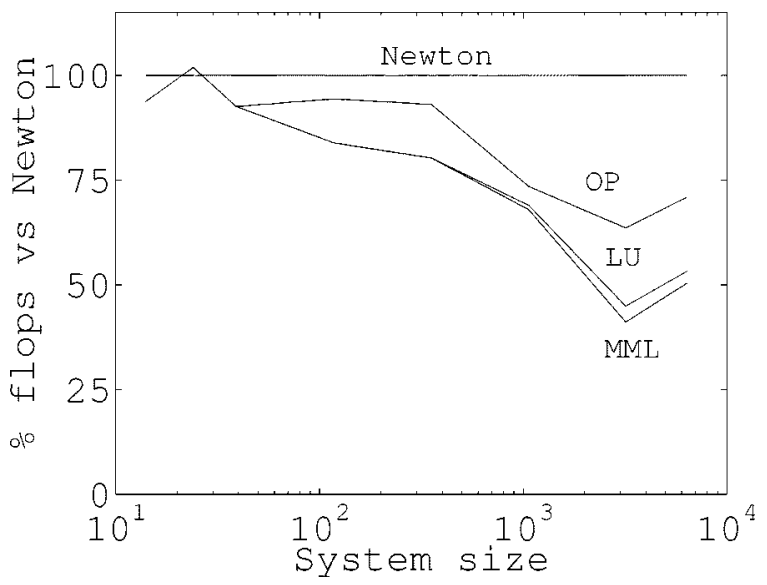

Fig. 9. Percentage of number of flops vs. Newton.

close to 50\%; g) When MML is used as a final step, after one or more LU update steps, we save another $2 \%$ to $10 \%$ in large systems. The largest savings (in percentage) occur with the large systems. For example for the 1062 bus system we save $32 \%$, for the 3186 bus system we save 59\%, and for the 6372 bus system we save $49 \%$.

It is well known that the convergence properties of the NPF depend, among other physical system characteristics, on the loading conditions and the location of the slack bus. We have performed a considerable number of experiments overloading certain buses and changing widely the location of the slack bus and observed that our method successfully detected the laggards. For those difficult cases the NPF requires more iterations and thus our QNPF becomes comparatively more efficient.

\section{Concluding Guidelines}

As general guidelines we recommend: a) For systems smaller than 1000 buses with no special loading conditions, the classical
Newton's method; b) For large systems and high accuracy requirements, the use of the proposed strategy with no PJU updates; c) For large systems, when the laggard residuals are identifiable and for practical accuracy, PJU with LU updates should be included. Also this approach is recommended for voltage stability studies even for small systems when one or two buses are overloaded; d) When the residuals cannot be identified early in the iteration process or when the method has not converged in 7 or 8 iterations because of a few laggards, a PJU step with MML correction is recommended.

\section{CONCLUSION}

We have presented a quasi-Newton methodology that combines Newton steps with simple steps and partial Jacobian updates to obtain a very efficient solution of the power flow problem. The methodology includes the possibility of selecting the next best step based on an estimation of the residuals. Partial Jacobian Updates are included in the quasi-Newton power flow using LU factorization updates and/or the Matrix Modification Lemma. The method has been tested with several systems ranging in size from 14 to 6372 buses. For large power systems, we have obtained savings in the order of $50 \%$ compared to Newton's method.

\section{APPENDIX I}

Convergence of A Single EQuation Using Newton's METHOD

In the vicinity of its solution, an equation looks (in general) like:

$$
f(x)=x+\theta x^{2}=0
$$


Linearizing (A.1) around $x_{k}$ gives

$$
\begin{aligned}
f\left(x_{k+1}\right) & =f\left(x_{k}\right)+f^{\prime}\left(x_{k}\right) \Delta x \\
& =x_{k}+\theta x_{k}^{2}+\left(1+2 \theta x_{k}\right)\left(x_{k+1}-x_{k}\right)=0
\end{aligned}
$$

so that, as $x_{k} \rightarrow 0$,

$$
x_{k+1}=x_{k}-\frac{x_{k}+\theta x_{k}^{2}}{1+2 \theta x_{k}}=\frac{\theta x_{k}^{2}}{1+2 \theta x_{k}} \approx \theta x_{k}^{2} .
$$

\section{APPENDIX II}

\section{QUADRATIC CONVERGENCE OF THE JACOBIAN MATRIX}

We linearize $J(x)$ around $x_{k}$ (in fact, we linearize the column vector built from the columns of $J$ ):

$$
J_{k+1}-J_{k}=\mathrm{D} J_{k}\left(x_{k+1}-x_{k}\right)
$$

were $\mathrm{D} J_{k}$ is the "Jacobian" of $J$. Thus the variation of $J$ is closely proportional to $\Delta x$ or $b$; see (1).

\section{APPENDIX III ITERATION MATRIX}

When we solve

$$
A x=b
$$

using an approximation matrix $B$ for $A$ we have

$$
B \Delta x=\eta
$$

where the residual (or mismatch) $\eta$ at step $k$ is

$$
\eta_{k}=b-A x_{k}
$$

We obtain the convergence rate of the errors by substituting (A.7) into (A.6) and using $\Delta x=x_{k+1}-x_{k}$ :

$$
x_{k+1}=x_{k}+B^{-1}\left(b-A x_{k}\right) .
$$

Let $x_{\infty}$ be the converged solution of (A.5) and $\varepsilon_{k}$ the error such that

$$
x_{k}=x_{\infty}+\varepsilon_{k}, \quad x_{k+1}=x_{\infty}+\varepsilon_{k+1} .
$$

Substituting (A.9) into (A.8) yields

$$
\varepsilon_{k+1}=\left(I-B^{-1} A\right) \varepsilon_{k}=M_{\varepsilon} \varepsilon_{k} .
$$

Equation (A.10) gives the convergence rate for errors. For mismatches we start by arranging (A.7) as

$$
x_{k}=A^{-1}\left(b-\eta_{k}\right), \quad x_{k+1}=A^{-1}\left(b-\eta_{k+1}\right) .
$$

Substituting (A.11) into (A.8) and simplifying we find that the convergence rate for mismatches is given by

$$
\eta_{k+1}=\left(I-A B^{-1}\right) \eta_{k}=M_{\eta} \eta_{k}
$$

\section{APPENDIX IV \\ PARTIAL JACOBIAN UPDATES VIA MML}

We assume that we have computed the solution of a simple step

$$
B x_{o l d}=b
$$

where $B$ represents the constant Jacobian. We want to update $B$ by modifying only $p$ rows. The new (partially updated) Jacobian is

$$
A=B+U V
$$

were $A$ and $B$ are $n \times n$ matrices and $U$ and $V^{T}$ are $n \times p$ matrices. This is a rank- $p$ update where for the method to be efficient $p \ll n$. The matrix inversion lemma gives

$$
A^{-1}=B^{-1}-B^{-1} U K^{-1} V B^{-1}
$$

where $K=I+V B^{-1} U$. Thus, the solution of the new equation

$$
A x=b
$$

is

$$
x=A^{-1} b=x_{o l d}-B^{-1} U K^{-1} V x_{o l d} .
$$

We note that only matrix $K$ of the small size $p \times p$ needs to be factorized. All other computations are obtained by simple matrix-vector multiplications or by forward and backward substitutions.

\section{REFERENCES}

[1] B. Stott and O. Alsaç, "Fast decoupled load flow," IEEE Trans. Power Apparatus and Systems, vol. PAS-93, no. 4, pp. 859-869, May/June 1974.

[2] W. F. Tinney and C. E. Hart, "Power flow solution by Newton's method," IEEE Trans. Power Apparatus and Systems, vol. PAS-86, no. 11, pp. 1449-1460, Nov. 1967.

[3] A. J. Flueck and H. D. Chiang, "Solving the nonlinear power flow equations with an inexact Newton method using GMRES," IEEE Trans. Power Systems, vol. 13, no. 2, pp. 267-273, May 1998.

[4] M. E. El-Hawary, Electrical Power Systems, ser. Power Engineering Series: IEEE Press, 1995, pp. 328-334.

[5] A. Jennings, Matrix Computation for Engineers and Scientists: John Wiley \& Sons, Ltd., 1977, p. 189.

[6] S. Chan and V. Brandwajn, "Partial matrix refactorization," IEEE Trans. Power Systems, vol. PWRS-1, no. 1, pp. 193-200, Feb. 1986.

[7] A. Monticelli, A. Garcia, and O. R. Saavedra, "Fast decoupled load flow: Hypotesis, derivations, and testing," IEEE Trans. Power Systems, vol. 5, no. 4, pp. 1425-1431, Nov. 1990.

[8] R. Bacher and W. F. Tinney, "Faster local power flow solutions: The zero mismatch approach," IEEE Trans. Power Systems, vol. 4, no. 4, pp. 1345-1354, Oct. 1989

[9] M. La Scala, G. Sblendorio, A. Bose, and J. Q. Wu, "Comparison of algorithms for transient stability simulations on shared and distributed memory multiprocessors," IEEE Trans. Power Systems, vol. 8, no. 1, pp. 2045-2050, Nov. 1996.

Adam Semlyen was born in 1923 in Rumania where he received the Dipl. Ing and $\mathrm{Ph} . \mathrm{D}$. degrees. He started his career there with an electric power utility and held academic positions at the Polytechnic Institute of Timisoara. In 1969, he joined the University of Toronto where he is a professor in the Department of Electrical and Computer Engineering, emeritus since 1988. His research interests include steady state and dynamic analysis as well as computation of electromagnetic transients in power systems.

Francisco de León was born in Mexico City in 1959. He received the B.Sc. and M.Sc. degrees from Instituto Politécnico Nacional (IPN), Mexico, in 1983 and 1986, respectively. He received the Ph.D. degree in 1992 from the University of Toronto, Canada. From 1992 to 1998, he was with the Graduate Division of the IPN. He is currently working for Plitron Manufacturing Inc., designing toroidal transformers. His main research interests include the computation of electro-magnetic fields and transients in power apparatus and systems and the time domain modeling of nonlinear circuits. 